\title{
Disguised and new quasi-Newton methods for nonlinear eigenvalue problems
}

\author{
E. Jarlebring ${ }^{1}$ (D) A. Koskela ${ }^{1} \cdot$ G. Mele ${ }^{1}$
}

Received: 15 March 2017 / Accepted: 31 October 2017 / Published online: 21 November 2017 (C) The Author(s) 2017. This article is an open access publication

\begin{abstract}
In this paper, we take a quasi-Newton approach to nonlinear eigenvalue problems (NEPs) of the type $M(\lambda) v=0$, where $M: \mathbb{C} \rightarrow \mathbb{C}^{n \times n}$ is a holomorphic function. We investigate which types of approximations of the Jacobian matrix lead to competitive algorithms, and provide convergence theory. The convergence analysis is based on theory for quasi-Newton methods and Keldysh's theorem for NEPs. We derive new algorithms and also show that several well-established methods for NEPs can be interpreted as quasi-Newton methods, and thereby, we provide insight to their convergence behavior. In particular, we establish quasi-Newton interpretations of Neumaier's residual inverse iteration and Ruhe's method of successive linear problems.
\end{abstract}

Keywords Nonlinear eigenvalue problems · Inverse iteration - Iterative methods · Quasi-Newton methods

\section{Introduction}

One of the most common techniques to improve the convergence or efficiency of Newton's method for nonlinear systems of equations is to replace the Jacobian matrix with another matrix. Among these quasi-Newton method constructions, sometimes called inexact Newton methods, the most common variation is to keep the Jacobian matrix constant. A factorization of this matrix can be precomputed before carrying out the iterations which usually makes the linear solves in the iteration cheaper. This

E. Jarlebring

eliasj@kth.se

1 KTH Royal Institute of Technology, Stockholm, Sweden 
is beneficial, e.g., in situations where the problem stems from a discretization of a PDE, as the resulting system is often large and the Jacobian matrix sparse with a structure allowing a sparse LU factorization to be precomputed.

In this paper, we consider nonlinear eigenvalue problems (NEPs) of the type

$$
M(\lambda) v=0, \quad v \neq 0,
$$

where $M: \mathbb{C} \rightarrow \mathbb{C}^{n \times n}$. Various flavors of Newton's method appear in the literature (further discussed below) for this class of NEPs. Some of these methods do have the property that the matrix in the linear system to be solved in every iteration remains constant. However, these methods are in general not seen as Jacobian matrix modifications of Newton's method, but are often derived from quite different viewpoints. In this paper, we investigate methods resulting from modifying the Jacobian matrix of an associated augmented system in various ways and illustrate differences and similarities of the resulting methods in terms of theoretical properties and efficiency. It turns out that several well-established approaches for NEPs can be viewed as quasi-Newton methods applied on an augmented system.

Suppose $\Omega \subset \mathbb{C}$ is a closed set and $M$ analytic in $\Omega$. We call the vector $v$ a (right) eigenvector if it satisfies (1.1) for some $\lambda \in \Omega$ and correspondingly $u$ is called the left eigenvector if it satisfies

$$
u^{H} M(\lambda)=0, \quad u \neq 0 .
$$

We call $(\lambda, v, u)$ an eigentriplet of (1.1) corresponding to the eigenvalue $\lambda$. Without loss of generality, we phrase the NEP as a system of equations

$$
F\left(\left[\begin{array}{l}
v \\
\lambda
\end{array}\right]\right):=\left[\begin{array}{c}
M(\lambda) v \\
c^{H} v-1
\end{array}\right]=0
$$

which is equivalent to (1.1) if $c \in \mathbb{C}^{n}$ is not orthogonal to the eigenvector $v$. This condition is not problematic in practice since $c$ can be chosen freely and thus will generically not be orthogonal to any eigenvector. The quasi-Newton approach to (1.3) consists of generating sequences of approximations $\left(\mu_{1}, x_{1}\right),\left(\mu_{2}, x_{2}\right), \ldots$ from the update formula

$$
\tilde{J}_{k}\left[\begin{array}{c}
x_{k+1}-x_{k} \\
\mu_{k+1}-\mu_{k}
\end{array}\right]=-\left[\begin{array}{c}
M\left(\mu_{k}\right) x_{k} \\
c^{H} x_{k}-1
\end{array}\right]=-F_{k}
$$

where $\tilde{J}_{k}$ is an approximation of the Jacobian matrix

$$
\tilde{J}_{k} \approx J_{k}=J\left(\left[\begin{array}{l}
x_{k} \\
\mu_{k}
\end{array}\right]\right):=\left[\begin{array}{cc}
M\left(\mu_{k}\right) & M^{\prime}\left(\mu_{k}\right) x_{k} \\
c^{H} & 0
\end{array}\right] .
$$

The eigenvector and eigenvalue updates will be denoted $\Delta x_{k}=x_{k+1}-x_{k}$ and $\Delta \mu_{k}=$ $\mu_{k+1}-\mu_{k}$.

We consider four specific modifications of the Jacobian matrix, briefly justified as follows. From a quasi-Newton perspective, the most common approach consists of keeping the Jacobian matrix constant, i.e., setting

$$
\widetilde{J}_{1, k}:=\left[\begin{array}{cc}
M(\sigma) & M^{\prime}(\sigma) x_{0} \\
c^{H} & 0
\end{array}\right],
$$


where $\sigma=\lambda_{0}$ is the starting value for the eigenvalue and $x_{0}$ the starting vector for the eigenvector approximation. In this manuscript, we refer to this as quasi-Newton 1 (QN1). In Section 2.1, we show how (1.4) with Jacobian matrix approximation (1.6) can be reformulated such that in every iteration we need to solve one linear system associated with the matrix $M(\sigma)$.

We shall later show (in Section 2.1) that a more accurate approximation of the Jacobian matrix leads to an algorithm which has the same computational cost per iteration as QN1, i.e., it involves the solution of one linear system with the matrix $M(\sigma)$ per iteration. More precisely, we keep only the $(1,1)$-block constant by setting

$$
\widetilde{J}_{2, k}:=\left[\begin{array}{cc}
M(\sigma) & M^{\prime}\left(\mu_{k}\right) x_{k} \\
c^{H} & 0
\end{array}\right] .
$$

The quasi-Newton method (1.4) with Jacobian matrix approximation (1.7) will be referred to as quasi-Newton 2 (QN2).

We also investigate a method (which we call quasi-Newton 3 ) corresponding to keeping the $(1,1)$-block constant as in QN2, but also replace the derivative in the $(1,2)$-block. We replace the derivative with a finite difference involving the future eigenvalue approximation $\lambda_{k+1}$, i.e., we set

$$
\widetilde{J}_{3, k}:=\left[\begin{array}{cc}
M(\sigma) & M\left[\mu_{k+1}, \mu_{k}\right] x_{k} \\
c^{H} & 0
\end{array}\right],
$$

where we use the standard notation for divided differences

$$
M[\lambda, \mu]=\left\{\begin{array}{cc}
\frac{M(\lambda)-M(\mu)}{\lambda-\mu} & \lambda \neq \mu \\
M^{\prime}(\lambda) & \lambda=\mu .
\end{array}\right.
$$

Note that the modification of the Jacobian matrix in (1.8) makes the iteration (1.4) implicit in the sense that the formula (1.4) for the new approximation $\left(\lambda_{k+1}, v_{k+1}\right)$ involves $\lambda_{k+1}$ in a nonlinear way. Many implicit variations of Newton's method have been considered in the literature; see, e.g., $[12,18]$ and references therein. It turns out that certain implicit variations of Newton's method improve the convergence and sometimes even increase the convergence order. In contrast to many other implicit Newton methods, our choice of the Jacobian matrix is done with the goal of having a method whose iterates can be computed without solving a (computationally demanding) nonlinear system of equations. This is possible for the specific choice (1.8), as we shall illustrate in Section 2.

We consider one more modified Jacobian matrix which also leads to an implicit method, but now implicit in the eigenvector. The vector $x_{k}$ in the $(1,2)$ block is replaced by the future vector $x_{k+1}$, leading to the Jacobian approximation

$$
\widetilde{J}_{4, k}:=\left[\begin{array}{cc}
M\left(\mu_{k}\right) & M^{\prime}\left(\mu_{k}\right) x_{k+1} \\
c^{H} & 0
\end{array}\right] .
$$

Our study has the following conclusions and contributions:

- QN1 and QN2 can be phrased as algorithms only involving one linear solve with $M(\sigma)$ per iteration (Sections 2.1-2.2) 
- QN3 is equivalent to Neumaier's residual inverse iteration [25] (Section 2.3)

- QN4 is equivalent to Ruhe's method of successive linear problems [29] (Section 2.4)

- We provide exact characterizations of the convergence of factor for QN1 and QN2 and establish that the convergence factor of QN2 and QN3 is identical (Sections 3.1-3.2)

- We show how to adapt fundamental theory for inexact Newton method [5] to study QN4 (Section 3.3)

- We provide generalizations of convergence rate dependence on eigenvalue clustering analogous to methods for linear eigenvalue methods (Section 4)

The studied properties of the methods are illustrated in numerical simulations in Section 5.

Newton's method for linear and nonlinear eigenvalue problems has been studied for decades, and the field is still under active development, as can be observed in the summaries in $[22,40]$. The technique of deriving methods using an augmented system (1.3) was investigated already in 1950s by Unger [38] and was the key to characterizing the relationship of Newton's method and inverse iteration (for linear eigenvalue problems) as described by Peters and Wilkinson in [27]. Newton's method based on solving the nonlinear equation $\beta(\lambda)=0$ where $M(\lambda) v=\beta(\lambda) e_{p}$ was presented in [26] and independently by an essentially equivalent procedure by Lancaster [20]. In 1970s, Ruhe [29] also pointed out the relevance of Newton-type methods and how they relate to inverse iteration. He used the augmented system of equations to derive variations of inverse iteration for NEPs as well as the method of successive linear problems.

Newton-type methods have also more recently received considerable attention, e.g., in the PhD thesis of Schreiber [30], where two-sided generalizations of inverse iteration methods as well as Jacobi-Davidson methods are developed. The recent results by Effenberger and Kressner [7, 19] contain a generalization of Newton-type methods that allows the computation of several eigenvalues simultaneously. This block Newton approach has been successful in the setting of continuation methods [4]. Variants of Newton's methods where the linear system associated with $M(\sigma)$ is only solved to some accuracy have been studied in [34]. A recent variant of the rational Krylov method can also be interpreted in a Newton setting [1]. There are several convergence results for residual inverse iteration and other Newton-type methods $[17,34,35,37]$ which are mostly presented in a separated fashion without using quasi-Newton interpretations and results for quasi-Newton methods.

\section{Explicit reformulations of the quasi-Newton methods}

The formulations of the QN-iterations above are not practical in general. In particular, the formulations of QN3 and QN4 are not even iterative, since the Jacobian matrices depend on quantities in an implicit way. Nevertheless, it turns out that certain reformulations of QN3 and QN4 allow us to explicitly compute sequences of 
approximations which satisfy (1.4). Also, QN1 and QN2 have to be reformulated in order to become practical. We show how to carry out this reformulation to obtain algorithms which do not require solving many linear systems for different matrices, but only for $M(\sigma)$.

We first make an observation in common for all the considered methods. The last row of the correction (1.4) is the same for all methods and for all the choices of $\tilde{J}_{k}$ as in (1.6)-(1.10), we see that $c^{H}\left(x_{k+1}-x_{k}\right)=-c^{H} x_{k}+1$. By induction, this implies that

$$
c^{H} x_{1}=c^{H} x_{2}=\cdots=1 \text {, }
$$

i.e., all the iterates (except possibly the first iterate) are normalized.

\subsection{The $n$-dimensional form of QN1}

The iteration (1.4) with the Jacobian matrix approximation (1.6) can be reformulated as follows. By multiplying the first block equation in (1.4) with $M(\sigma)^{-1}$ from the left yields

$$
\Delta x_{k}+\Delta \mu_{k} M(\sigma)^{-1} M^{\prime}(\sigma) x_{0}=-M(\sigma)^{-1} M\left(\mu_{k}\right) x_{k}=-y_{k},
$$

where

$$
y_{k}:=M(\sigma)^{-1} M\left(\mu_{k}\right) x_{k} .
$$

Moreover, by multiplying (2.2) from the left with $c^{H}$ and using the fact that $c^{H} \Delta x_{k}=$ 0 due to (2.1), we have

$$
\mu_{k+1}-\mu_{k}=\Delta \mu_{k}=-\alpha_{0} c^{H} y_{k}
$$

with

$$
\begin{aligned}
q_{0} & :=M(\sigma)^{-1} M^{\prime}(\sigma) x_{0}, \\
\alpha_{0} & :=1 /\left(c^{H} q_{0}\right) .
\end{aligned}
$$

The above equations can be combined into an algorithm. As a precomputation, we compute $\alpha_{0}$ in (2.5b) and $q_{0}$ in (2.5a), and in the iteration, we compute $y_{k}$ from (2.3) and $\Delta \mu_{k}$ from (2.4) and subsequently update

$$
x_{k+1}=x_{k}-y_{k}-\Delta \mu_{k} q_{0} .
$$

The algorithm is summarized in Algorithm 1.

Remark 2.1 (Properties of Algorithm 1) The advantage of Algorithm 1 over the original formulation (1.4) consists in the fact that the matrix in the linear system to be solved in every step is $M(\sigma)$. Therefore, a prefactorization can be carried out in the original problem size. The factorization of $M(\sigma)$ is possible, e.g., when the problem stems from a partial differential equation, and it is of advantage as the augmented matrix $\tilde{J}_{1, k}$ may be more difficult to factorize. Although the Jacobian matrix approximation $J_{k} \approx \tilde{J}_{1, k}$ is the most common in the context of quasi-Newton 
approximations, it does not appear very competitive in this setting. The other Jacobian matrix approximations, which are more accurate, lead to better methods in terms of convergence and do not in general require more computation.

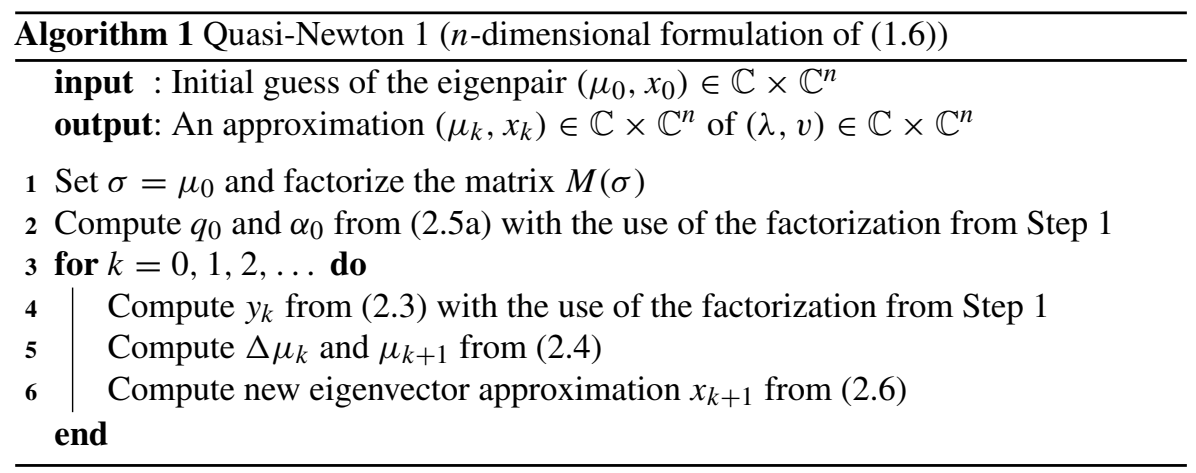

\subsection{The $n$-dimensional formulation of QN2}

A reformulation of (1.4) with the Jacobian matrix approximation (1.7) follows similar steps as the derivation in the previous section. To simplify the notation, we set

$$
w_{\sigma}^{H}:=c^{H} M(\sigma)^{-1} .
$$

The reformulation leads up to the formulas

$$
\begin{aligned}
\Delta \mu_{k} & =-\frac{w_{\sigma}^{H} M\left(\mu_{k}\right) x_{k}}{w_{\sigma}^{H} M^{\prime}\left(\mu_{k}\right) x_{k}} \\
z_{k} & =\Delta \mu_{k} M^{\prime}\left(\mu_{k}\right) x_{k}+M\left(\mu_{k}\right) x_{k} \\
\mu_{k+1} & =\mu_{k}+\Delta \mu_{k} \\
x_{k+1} & =x_{k}-M(\sigma)^{-1} z_{k} .
\end{aligned}
$$

More precisely, formula (2.8a) stems from left-multiplying the first block row in (1.4) with $w_{\sigma}^{H}=c^{H} M(\sigma)^{-1}$ and (2.8b) and (2.8d) stem from left-multiplying the first block row in (1.4) with $M(\sigma)^{-1}$. The resulting method is summarized in Algorithm 2.

Remark 2.2 (Properties of Algorithm 2) Similarly to Algorithm 1, Algorithm 2 requires only one linear solve with the matrix $M(\sigma)$ per iteration. Since Algorithm 2 corresponds to a more accurate approximation of the Jacobian matrix, it is expected to converge faster than Algorithm 1. This difference is characterized theoretically and computationally in Sections 3.1 and 5.

Algorithm 2 involves the vector $w_{\sigma}$, which can be computed as in (2.7), i.e., it would require one additional linear solve with $M(\sigma)^{H}$. This extra linear solve can however be avoided by treating $w_{\sigma}$ as a fixed vector (chosen by the user) and then choosing $c^{H}=w_{\sigma}^{T} M(\sigma)$. This works rather well in practice, but fixing $w_{\sigma}$ instead of $c$ may make the convergence factor larger if $\sigma$ is close to the eigenvalue, as we shall further illustrate in Section 4. 


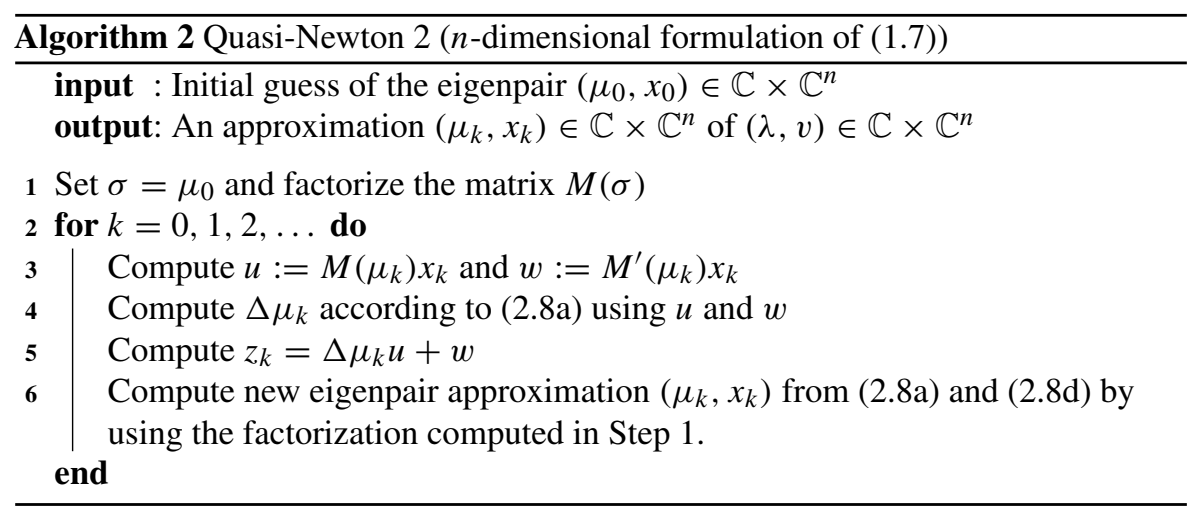

\subsection{The explicit formulation of QN3 is residual inverse iteration}

Although the Jacobi approximation $J_{k} \approx \tilde{J}_{3, k}$ in (1.8) involves an eigenvalue approximation not yet computed, we can proceed with an elimination procedure similar to Sections 2.1 and 2.2.

We first multiply the first block in (1.4) from the left with $w_{\sigma}^{H}=c^{H} M(\sigma)^{-1}$. This gives an equation which can be simplified to the following system of equations:

$$
\begin{aligned}
w_{\sigma}^{H} \frac{M\left(\mu_{k+1}\right)-M\left(\mu_{k}\right)}{\mu_{k+1}-\mu_{k}} x_{k} \Delta \mu_{k} & =-w_{\sigma}^{H} M\left(\mu_{k}\right) x_{k} \\
w_{\sigma}^{H} M\left(\mu_{k+1}\right) x_{k} & =0 .
\end{aligned}
$$

Note that (2.9b) is a scalar-valued equation in the unknown variable $\mu_{k+1}$, since $x_{k}$ can be viewed as a known vector. In fact, if $\lambda$ is treated as a function of $x$, it can be viewed as the function implicitly defined by $w^{H} M(\lambda) x=0$, which is commonly known as the Rayleigh functional or generalized Rayleigh quotient [39, 41, 42]. This function generally exists, at least in a neighborhood of a simple eigenvalue [17, Proposition 2.1], and it is a computable quantity for many problems.

By multiplying the first block row in (1.4) from the left by $M(\sigma)^{-1}$, we obtain

$$
\Delta x_{k}+M(\sigma)^{-1}\left(M\left(\mu_{k+1}\right)-M\left(\mu_{k}\right)\right) x_{k}=-M\left(\mu_{k}\right) x_{k}
$$

which can be simplified as

$$
x_{k+1}=x_{k}-M(\sigma)^{-1} M\left(\mu_{k+1}\right) x_{k} .
$$

Under the assumption that the Rayleigh functional in (2.9b) is computable, the relations (2.9b) and (2.10) form an explicit algorithm. The resulting method is summarized in Algorithm 3. Due to the choice of $w_{\sigma}$, the normalization property $c^{T} x_{k}=1$ is satisfied for all $k$ in theory, but can be lost in practice due to finite arithmetic. Therefore, we have added an explicit normalization step in the algorithm.

In fact, this algorithm is already extensively used in current research, where it is commonly known as residual inverse iteration and it was first introduced by Neumaier in [25]. Residual inverse iteration also forms the basis of some recent state-of-the-art algorithms for NEPs, most importantly the nonlinear Arnoldi method [39]. 
Theorem 2.1 The quasi-Newton method (1.4) with the modified Jacobian matrix (1.8) is equivalent to residual inverse iteration as described in [25].

Remark 2.3 (Relation between quasi-Newton variant 2 and residual inverse iteration) Due to the analyticity of $M(\lambda)$, residual inverse iteration defined by (2.9b) and (2.10) can also be expressed as

$$
\begin{aligned}
& \mu_{k+1}=\mu_{k}-\frac{w^{H} M\left(\mu_{k}\right) v_{k}}{w^{H} M^{\prime}\left(\mu_{k}\right) v_{k}}-\sum_{j=2}^{\infty} \frac{\Delta \mu_{k}^{j}}{j !} \frac{w^{H} M^{(j)}\left(\mu_{k}\right) v_{k}}{w^{H} M^{\prime}\left(\mu_{k}\right) v_{k}} \\
& v_{k+1}=v_{k}-M(\sigma)^{-1}\left[M\left(\mu_{k}\right)+\Delta \mu_{k} M^{\prime}\left(\mu_{k}\right)\right] v_{k}-\sum_{j=2}^{\infty} \frac{\Delta \mu_{k}^{j}}{j !} M(\sigma)^{-1} M^{(j)}\left(\mu_{k}\right) v_{k} .
\end{aligned}
$$

From these formulas, we directly see that for linear eigenvalue problems where $M(\lambda)=A-\lambda I, \mathrm{QN} 2$ and residual inverse iteration are equivalent. Hence, they are both generalizations of the standard inverse iteration method. This is consistent with the convergence analysis of Section 3 which shows that QN2 and QN3 have the same convergence factor.

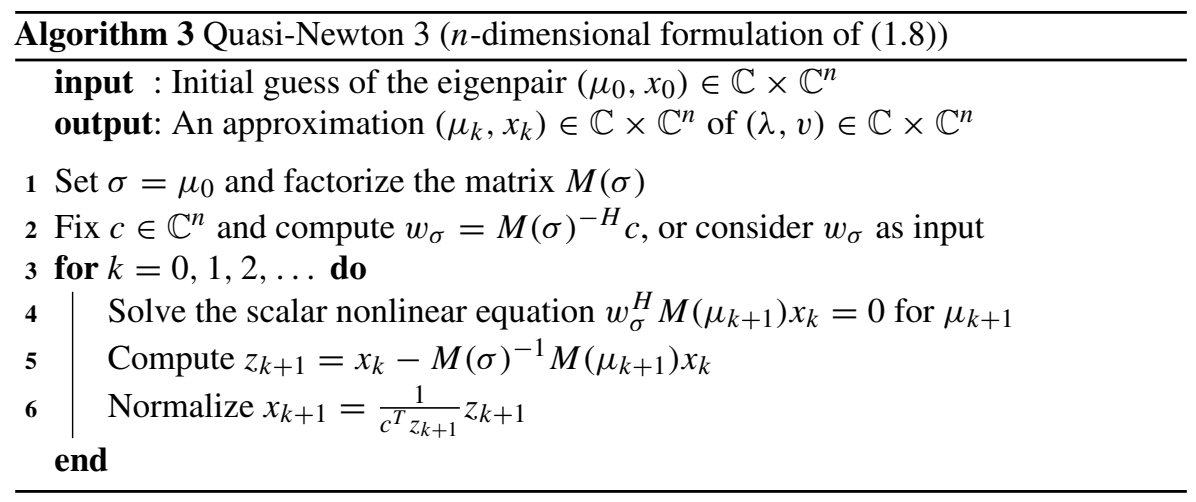

\subsection{The explicit formulation of QN4 is the method of successive linear problems}

In the previous subsection, we saw that iterates satisfying (1.4) with the modified Jacobian matrix (1.8) can be computed in practice and the resulting algorithm is in fact equivalent to a well-known method. The Jacobi approximation $J_{k} \approx \tilde{J}_{4, k}$ in (1.10) also involves a quantity which we do not have an access to at iteration $k$, the vector $x_{k+1}$. We now show that similarly as for QN3, we can carry out an elimination such that the update can be computed in an explicit way. This algorithm also turns out to be equivalent to a well-established method.

The first block row in (1.4) with the approximation (1.10) simplifies to

$$
M\left(\mu_{k}\right)\left(x_{k+1}-x_{k}\right)+\Delta \mu_{k} M^{\prime}\left(\mu_{k}\right) x_{k+1}=-M\left(\mu_{k}\right) x_{k}
$$

which can be further simplified to

$$
M\left(\mu_{k}\right) x_{k+1}+\Delta \mu_{k} M^{\prime}\left(\mu_{k}\right) x_{k+1}=0 .
$$


Since we know that the iterates $x_{1}, x_{2}, \ldots$ are normalized, we directly identify (2.11) as a (linear) generalized eigenvalue problem where $\Delta \mu_{k}$ is the eigenvalue. Hence, we can construct an iteration satisfying (1.4) with Jacobi approximation (1.10) by repeatedly solving the generalized eigenvalue problem (2.11) and updating the eigenvalue $\mu_{k+1}=\mu_{k}+\Delta \mu_{k}$. The resulting method is summarized in Algorithm 4. This method is known as the method of successive linear problems and was studied and used by Ruhe in [29], where it was justified directly from a Taylor expansion of $M(\lambda)$.

Theorem 2.2 The quasi-Newton method (1.4) with the modified Jacobian matrix (1.10) is equivalent to the method of successive linear problems [29].

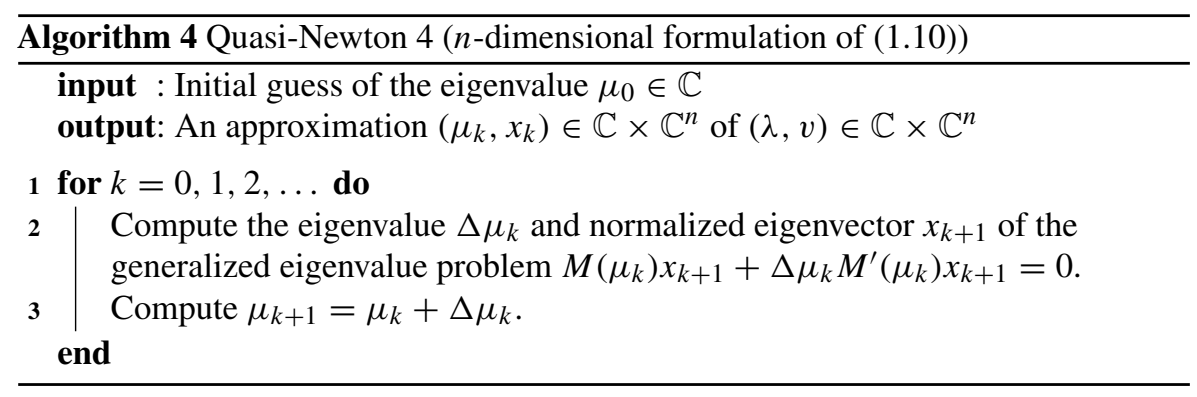

\section{Local convergence analysis}

\subsection{Convergence factor analysis of QN1 and QN2}

In order to characterize the convergence of QN1 and QN2, we will derive a firstorder result. More precisely, for a pair $\left(x_{k}, \mu_{k}\right)$ close to $(\lambda, v)$, we describe the local behavior with a matrix $A \in \mathbb{C}^{(n+1) \times(n+1)}$ such that

$$
\left[\begin{array}{l}
x_{k+1}-v \\
\mu_{k+1}-\lambda
\end{array}\right]=A\left[\begin{array}{l}
x_{k}-v \\
\mu_{k}-\lambda
\end{array}\right]+\mathcal{O}\left(\left\|\left[\begin{array}{c}
x_{k}-v \\
\mu_{k}-\lambda
\end{array}\right]\right\|^{2}\right)
$$

In general, the quasi-Newton methods considered here give a linear convergence, with a local convergence factor given by the spectral radius of $A$. The explicit form of $A$ for our first two quasi-Newton methods is given in the following two theorems.

Theorem 3.1 (Local convergence Algorithm 1) Suppose the sequence $\left(\mu_{1}, x_{1}\right)$, $\left(\mu_{2}, x_{2}\right), \ldots$ is generated by Algorithm 1 started with $\left(\mu_{0}, x_{0}\right)$ and suppose the sequence converges to the eigenpair $(\lambda, v)$. Then, the sequence satisfies (3.1) with $A=A_{1}$ where

$$
A_{1}=\left[\begin{array}{cc}
\left(I-\alpha_{0} q_{0} c^{H}\right) M(\sigma)^{-1}(M(\sigma)-M(\lambda)) & \left(I-\alpha_{0} q_{0} c^{H}\right) M(\sigma)^{-1} M^{\prime}(\lambda) v \\
\alpha_{0} c^{H} M(\sigma)^{-1}(M(\sigma)-M(\lambda)) & \alpha_{0} c^{H} M(\sigma)^{-1} M^{\prime}(\lambda) v
\end{array}\right] .
$$


Proof In a fixed point setting, QN1 can be expressed as

$$
\begin{aligned}
{\left[\begin{array}{l}
v_{k+1} \\
\lambda_{k+1}
\end{array}\right] } & =\varphi\left(\left[\begin{array}{l}
v_{k} \\
\lambda_{k}
\end{array}\right]\right)=\left[\begin{array}{l}
v_{k} \\
\lambda_{k}
\end{array}\right]-\left[\begin{array}{cc}
M(\sigma) & M^{\prime}(\sigma) x_{0} \\
c^{H} & 0
\end{array}\right]^{-1} F\left(\left[\begin{array}{l}
v_{k} \\
\lambda_{k}
\end{array}\right]\right) \\
& =\left[\begin{array}{l}
v_{k} \\
\lambda_{k}
\end{array}\right]-\tilde{J}_{1, k}^{-1} F\left(\left[\begin{array}{l}
v_{k} \\
\lambda_{k}
\end{array}\right]\right),
\end{aligned}
$$

where $\tilde{J}_{1, k}$ is given as in (1.6). (Although $\tilde{J}_{1, k}$ is constant with respect to $k$, we will not explicitly use this since this allows us to use analogous reasoning in the proof Theorem 3.2.)

The $A$-matrix in (3.1), corresponding to a fixed point map, is given by the Jacobian of $\varphi$. Note that the Jacobian of $F$ is given by

$$
J_{*}:=\left[\begin{array}{cc}
M(\lambda) & M^{\prime}(\lambda) v \\
c^{H} & 0
\end{array}\right] .
$$

By application of the product rule for differentiation and using that $F\left(\left[\begin{array}{l}v \\ \lambda\end{array}\right]\right)=0$, we have

$$
\begin{aligned}
A & =\varphi^{\prime}\left(\left[\begin{array}{l}
v \\
\lambda
\end{array}\right]\right)=I-\tilde{J}_{1, *}^{-1} J_{*}=\tilde{J}_{1, *}^{-1}\left(\tilde{J}_{1, *}-J_{*}\right) \\
& =\left[\begin{array}{cc}
M(\sigma) & M^{\prime}(\sigma) x_{0} \\
c^{H} & 0
\end{array}\right]^{-1}\left[\begin{array}{cc}
M(\sigma)-M(\lambda) & M^{\prime}(\sigma) x_{0}-M^{\prime}(\lambda) v \\
0 & 0
\end{array}\right],
\end{aligned}
$$

where $\tilde{J}_{1, *}$ denotes $\tilde{J}_{1, k}$ in (1.6) evaluated in the solution. The $A$-matrix in (3.2) follows from (3.4) after the application of the Schur complement formula for $\tilde{J}_{1, *}^{-1}$.

Theorem 3.2 (Local convergence Algorithm 2) Suppose the sequence $\left(\mu_{1}, x_{1}\right)$, $\left(\mu_{2}, x_{2}\right), \ldots$ is generated by Algorithm 1 started with $\left(\mu_{0}, x_{0}\right)$ and suppose the sequence converges to the eigenpair $(\lambda, v)$. Then, the sequence satisfies (3.1) with $A=A_{2}$ where

$$
A_{2}=\left[\begin{array}{cc}
\left(I-\alpha q c^{H}\right) M(\sigma)^{-1}[M(\sigma)-M(\lambda)] & 0 \\
\alpha c^{H} M(\sigma)^{-1}(M(\sigma)-M(\lambda)) & 0
\end{array}\right] .
$$

where $\alpha:=\left(w_{\sigma}^{H} M^{\prime}(\lambda) v\right)^{-1}$ and $q:=M(\sigma)^{-1} M^{\prime}(\lambda) v$.

Proof The proof follows the same reasoning as the proof of Theorem 3.1, except that the Jacobian matrix of the fixed point map $\varphi$ in (3.4) in this case becomes

$$
A=\left[\begin{array}{cc}
M(\sigma) & M^{\prime}(\lambda) x \\
c^{H} & 0
\end{array}\right]^{-1}\left[\begin{array}{cc}
M(\sigma)-M(\lambda) & 0 \\
0 & 0
\end{array}\right] .
$$

The application of the Schur complement formula directly leads to (3.5).

\subsection{Local convergence of QN3}

Note that QN3 is not a fixed point iteration in the formulation (1.8). However, since residual inverse iteration and QN3 are equivalent in the sense of Theorem 2.1, we 
already have a convergence factor available in [17]. More surprisingly, the convergence factor for residual inverse iteration (given in [17]) is identical to the convergence factor of QN2.

Corollary 3.3 (Convergence factor equivalence of QN2 and QN3) The non-zero eigenvalues of the matrix $A_{2}$ given in (3.5) are the same as the non-zero eigenvalues of

$$
B=\left(I-v c^{H}\right) M(\sigma)^{-1}\left[M(\sigma)-M(\lambda)+\frac{1}{w_{\sigma}^{H} M^{\prime}(\lambda) v} M^{\prime}(\lambda) v w_{\sigma}^{H} M(\lambda)\right]
$$

and the convergence factors of QN2 and QN3 are the same.

Proof Since QN3 is equivalent to residual inverse iteration according to Theorem 2.1, we can directly use the convergence characterization given in [17]. More precisely, [17, Theorem 3.1] states that the convergence factor of residual inverse iteration is the largest eigenvalue of the matrix $B$ in (3.6).

It remains to show that the non-zero eigenvalues of $B$ are the same as the non-zero eigenvalues of $A_{2}$ given in (3.5). Clearly, the non-zero eigenvalues of $A_{2}$ are the nonzero eigenvalues of the $(1,1)$-block of $A_{2}$. The equivalence is based on the general property that if a matrix $C$ and vectors $c$ and $v$ satisfy $c^{H} C=0$ and $c^{H} v=1$, then $C$ and $C\left(I-v c^{H}\right)$ have the same non-zero eigenvalues. This can be seen from the fact that if $\gamma_{i} z_{i}=C z_{i}$, then $\gamma_{i} c^{H} z_{i}={ }^{H} C z_{i}=0$ and $c^{H} z_{i}=0$ if $\gamma_{i} \neq 0$, i.e., $C\left(I-v c^{H}\right) z_{i}=C z_{i}-v c^{H} z_{i}=\gamma_{i} z_{i}$. By using this general property where $C$ is the (1,1)-block of (3.5), we obtain $B=C\left(I-v c^{H}\right)$ where $B$ is given in (3.6).

\subsection{Local convergence of QN4}

The quasi-Newton method corresponding to (1.10) is different in character in comparison to the other quasi-Newton methods we have considered. This method does not involve the computation of a linear system for a constant matrix. Although some convergence results for the method of successive linear problems (and therefore also QN4) are available in the literature [15], it is natural in our quasi-Newton approach to characterize the convergence using general results for quasi-Newton methods. It turns out that (1.10) is generally a very accurate approximation of the Jacobian matrix and we can apply results for quasi-Newton methods given in [5].

The characterization in [5] is mainly based on a quantity which describes the inexactness of the quasi-Newton method by comparing it with a step involving the exact Jacobian matrix. More precisely, we consider the vector $r_{k}$ in [5], which in our context becomes

$$
r_{k}:=J_{k}\left[\begin{array}{c}
x_{k+1}-x_{k} \\
\mu_{k+1}-\mu_{k}
\end{array}\right]+\left[\begin{array}{c}
M\left(\mu_{k}\right) x_{k} \\
c^{H} x_{k}-1
\end{array}\right]=\left[\begin{array}{c}
\left(\mu_{k+1}-\mu_{k}\right) M^{\prime}\left(\mu_{k}\right)\left(x_{k+1}-x_{k}\right) \\
0
\end{array}\right] .
$$

Various results are given in [5] in terms of the norm of $r_{k}$. In particular, the result [5, Theorem 3.3] demonstrates that we have a local quadratic convergence if $\left\|r_{k}\right\|=$ 
$\mathcal{O}\left(\left\|F_{k}\right\|^{2}\right)$. Due to the fact that all iterates are normalized as in (2.1), this condition can be simplified in our case and stated as

$$
\left\|r_{k}\right\|=\mathcal{O}\left(\left\|F\left(\left[\begin{array}{l}
x_{k} \\
\mu_{k}
\end{array}\right]\right)\right\|^{2}\right)=\mathcal{O}\left(\left\|M\left(\mu_{k}\right) x_{k}\right\|^{2}\right) .
$$

In order to use this result, we now assume that the Jacobian matrix in the eigenpair denoted $J_{*}$ and defined by (3.3) is invertible. This assumption holds in particular if the eigenpair $(\lambda, v)$ is such that $c^{H} v \neq 0$ and the eigenvalue $\lambda$ is simple. This implies that the function $F: \mathbb{C}^{n+1} \rightarrow \mathbb{C}^{n+1}$ is invertible in a neighborhood of $(\lambda, v)$. If $\left(\mu_{k}, x_{k}\right)$ is in this neighborhood, we have from the implicit function theorem that

$$
\left[\begin{array}{l}
x_{k}-v \\
\mu_{k}-\lambda
\end{array}\right]=-J_{*}^{-1}\left[\begin{array}{c}
M\left(\mu_{k}\right) x_{k} \\
0
\end{array}\right]+\mathcal{O}\left(\left\|M\left(\mu_{k}\right) x_{k}\right\|^{2}\right) .
$$

Theorem 3.4 (Convergence QN4) Suppose $\left(\mu_{1}, x_{1}\right),\left(\mu_{2}, x_{2}\right), \ldots$ are iterates satisfying (1.10) and suppose they converge to the eigenpair $(\lambda, v)$. If $(\lambda, v)$ is a simple or semisimple eigenpair, then $\left(\mu_{k}, x_{k}\right)$ converges at least quadratically to $(\lambda, v)$.

Proof From (3.7) and properties of the two norms, we have

$$
\begin{aligned}
\left\|r_{k}\right\| & \leq\left|\mu_{k+1}-\mu_{k}\right|\left\|M^{\prime}\left(\mu_{k}\right)\right\|\left\|x_{k+1}-x_{k}\right\| \\
& \leq\left(\left\|\left[\begin{array}{l}
x_{k+1}-v \\
\mu_{k+1}-\lambda
\end{array}\right]-\left[\begin{array}{c}
x_{k}-v \\
\mu_{k}-\lambda
\end{array}\right]\right\|\right)\left\|M^{\prime}\left(\mu_{k}\right)\right\|\left(\left\|\left[\begin{array}{l}
x_{k+1}-v \\
\mu_{k+1}-\lambda
\end{array}\right]-\left[\begin{array}{c}
x_{k}-v \\
\mu_{k}-\lambda
\end{array}\right]\right\|\right) \\
& \leq 4\left\|\left[\begin{array}{l}
x_{k}-v \\
\mu_{k}-\lambda
\end{array}\right]\right\|^{2}\left\|M^{\prime}\left(\mu_{k}\right)\right\| .
\end{aligned}
$$

In the last step, we used the assumption that $\left(\mu_{1}, x_{1}\right),\left(\mu_{2}, x_{2}\right), \ldots$ converges monotonically to an eigenpair, i.e.,

$$
\left\|\left[\begin{array}{c}
x_{k+1}-v \\
\mu_{k+1}-\lambda
\end{array}\right]\right\| \leq\left\|\left[\begin{array}{c}
x_{k}-v \\
\mu_{k}-\lambda
\end{array}\right]\right\|,
$$

for sufficiently large $k$. By using the expansion (3.8), we find directly that $\left\|r_{k}\right\|=$ $\mathcal{O}\left(\left\|M\left(\mu_{k}\right) x_{k}\right\|^{2}\right)$, which by [5, Theorem 3.3] implies quadratic convergence.

\section{Interpretation of convergence factors}

In order to provide further insight into the convergence behavior of the considered methods, we now make characterizations of the coefficient matrix $A$ in the fixed point formulation. To this end, we use results associated with (what is commonly referred to as) Keldysh's theorem; see the general formulation in [23] and the more recent descriptions in the context of NEPs, e.g. [3, 34]. For simple eigenvalues, Keldysh's theorem implies that there exists a function $R_{1}(\sigma)$, analytic in a neighborhood of the eigenvalue, such that

$$
M(\sigma)^{-1}=\frac{1}{\sigma-\lambda_{1}} \frac{v_{1} u_{1}^{H}}{u_{1}^{H} M^{\prime}\left(\lambda_{1}\right) v_{1}}+R_{1}(\sigma) \text { for all } \sigma \in \Omega \backslash\left\{\lambda_{1}\right\},
$$


where $\left(\lambda_{1}, v_{1}, u_{1}\right)$ is the eigentriplet of a simple eigenvalue.

We first observe that the convergence factor of QN2 and QN3 can directly be analyzed by using Keldysh's theorem, since for the spectral radius $\rho\left(A_{2}\right)$, it holds

$$
\begin{aligned}
\rho\left(A_{2}\right) \leq & \left\|\left(I-v_{1} c^{H}\right) M(\sigma)^{-1}\left[M(\sigma)-M\left(\lambda_{1}\right)+\frac{M^{\prime}\left(\lambda_{1}\right) v_{1} w_{\sigma}^{H} M\left(\lambda_{1}\right)}{w_{\sigma}^{H} M^{\prime}\left(\lambda_{1}\right) v_{1}}\right]\right\| \\
\leq & \left|\lambda_{1}-\sigma\right|\left\|\left(I-v_{1} c^{H}\right) R_{1}(\sigma)\left(M^{\prime}\left(\lambda_{1}\right)+M^{\prime}\left(\lambda_{1}\right) v_{1} c^{H} R_{1}\left(\lambda_{1}\right) M\left(\lambda_{1}\right)\right)\right\| \\
& +\mathcal{O}\left(\left|\lambda_{1}-\sigma\right|^{2}\right) .
\end{aligned}
$$

In the last inequality, we used that

$$
\lim _{\sigma \rightarrow \lambda_{1}} \frac{1}{\sigma-\lambda_{1}} \frac{M^{\prime}\left(\lambda_{1}\right) v_{1}}{w_{\sigma}^{H} M^{\prime}\left(\lambda_{1}\right) v} w_{\sigma}^{H} M\left(\lambda_{1}\right)=M^{\prime}\left(\lambda_{1}\right) v_{1} c^{H} R_{1}\left(\lambda_{1}\right) M\left(\lambda_{1}\right)
$$

which follows from (4.1). The relationship (4.2) indicates that the convergence factor depends linearly on the shift-eigenvalue distance, and linearly in $M^{\prime}\left(\lambda_{1}\right)$.

Remark 4.1 (Double non-semisimple eigenvalues) In case the eigenvalue $\lambda_{1}$ is a double non-semisimple eigenvalue with right and left eigenvectors $v$ and $u$, there exist the so-called generalized eigenvectors $\widetilde{v}$ and $\tilde{u}$ such that

$$
\begin{aligned}
M^{\prime}\left(\lambda_{1}\right) v+M\left(\lambda_{1}\right) \tilde{v} & =0 \\
u^{*} M^{\prime}\left(\lambda_{1}\right)+\tilde{u}^{*} M\left(\lambda_{1}\right) & =0 .
\end{aligned}
$$

where $u$ and $v$ are the left and right eigenvectors corresponding to $\lambda_{1}$. According to [3, Theorem 2.6] with $L=1$ and $m_{1}=2$, there is a neighborhood $U$ of $\lambda_{1}$ where we have the expansion

$$
M(\sigma)^{-1}=\frac{1}{u^{*} M^{\prime}\left(\lambda_{1}\right) \tilde{v}+\frac{1}{2} u^{*} M^{\prime \prime}\left(\lambda_{1}\right) v}\left[\frac{1}{\sigma-\lambda_{1}}\left(v \tilde{u}^{*}+\widetilde{v} u^{*}\right)+\frac{1}{\left(\sigma-\lambda_{1}\right)^{2}} v u^{*}\right]+R(\sigma),
$$

where $R(\sigma)$ is analytic in $U$. Then, instead of (3.5) the iteration matrix $A_{2}$ is of the form

$$
\begin{aligned}
A_{2}= & \left(I-v c^{T}\right)\left(\frac{1}{\sigma-\lambda_{1}} \frac{\tilde{v} u^{T}}{u^{*} M^{\prime}\left(\lambda_{1}\right) \widetilde{v}+\frac{1}{2} u^{*} M^{\prime \prime}\left(\lambda_{1}\right) v}+R(\sigma)\right)\left[M(\sigma)-M\left(\lambda_{1}\right)\right] \\
& +\mathcal{O}\left(\left|\lambda_{1}-\sigma\right|^{2}\right),
\end{aligned}
$$

where $R(\sigma)$ contains the contribution from all the eigenvalues other than $\lambda_{1}$. Thus, the iteration matrix $A-v c^{T}$ contains the factor $\frac{1}{\sigma-\lambda_{1}}$ and unlike in the case of a simple eigenvalue, the convergence factor is not asymptotically proportional to $\left|\lambda_{1}-\sigma\right|$.

\subsection{Eigenvalue clustering and condition number}

The convergence factor bound (4.2) provides insight on how the convergence depends on the shift if the shift-eigenvalue distance is small (consistent with what was pointed out in [17]). We now show that further insight can be provided by using a more general form of Keldysh's theorem. This applies to the situation where the shifteigenvalue distance is not necessarily small. 
Inverse iteration for linear eigenvalue problems (with the normalization $c^{H} v=1$ ) has the following property for diagonalizable matrices. The convergence factor for the eigentriplet $\left(\lambda_{1}, v_{1}, u_{1}\right)$ can be bounded in terms of reciprocal eigenvalue-shift distances weighted with the condition numbers as

$$
\rho\left(\left(I-v_{1} c^{H}\right) \sum_{i=2}^{n} \frac{\sigma-\lambda_{1}}{\sigma-\lambda_{i}} \frac{v_{i} u_{i}^{H}}{u_{i}^{H} v_{i}}\right) \leq\left\|P_{1}\right\|\left|\sigma-\lambda_{1}\right| \sum_{i=2}^{n} \frac{1}{\left|\sigma-\lambda_{i}\right|} \kappa_{i},
$$

where $\kappa_{i}=\left\|u_{i}\right\|\left\|v_{i}\right\| /\left|u_{i}^{H} v_{i}\right|$ is the eigenvalue condition number (following the standard definition [21]) and $P_{1}$ is the projector $P_{1}=I-v_{1} c^{H}$.

In order to generalize this property, we use the following form of Keldysh's theorem. We let $\Gamma \subset \Omega$ be a simple, closed, piecewise-smooth curve and denote the eigenvalues in its interior by $\lambda_{1}, \cdots, \lambda_{k}$. Then, Keldysh's theorem states that

$$
M(\sigma)^{-1}=\sum_{i=1}^{k} \frac{1}{\sigma-\lambda_{i}} \frac{v_{i} u_{i}^{H}}{u_{i}^{H} M^{\prime}\left(\lambda_{i}\right) v_{i}}+R_{\Gamma}(\sigma) \text { for all } \sigma \in \Omega \backslash\left\{\lambda_{1}, \ldots, \lambda_{k}\right\},
$$

where $R_{\Gamma}$ is analytic in $\operatorname{int}(\Gamma)$. The following result provides an analog of the eigenvalue clustering property (4.4), under the assumption that $R_{\Gamma}(\sigma)$ is small.

Corollary 4.1 (Eigenvalue clustering) Suppose that $\Omega$ is a closed simply connected domain with a boundary $\Gamma$. Suppose that $M$ is analytic in this domain and that all the eigenvalues are simple. Denote the corresponding eigentriplets by $\left(\lambda_{1}, v_{1}, u_{1}\right), \ldots,\left(\lambda_{k}, v_{k}, u_{k}\right)$, with normalization $c^{H} v_{1}=\cdots=c^{H} v_{k}=1$. Then, the convergence factors for QN2 and QN3 are bounded as

$$
\begin{gathered}
\rho\left(A_{2}\right)=\rho\left(A_{3}\right) \leq\left\|P_{1}\right\| \\
\cdot\left(\sum_{i=2}^{k} \frac{1}{\left|\sigma-\lambda_{i}\right|} \kappa_{i}+\left\|R_{\Gamma}(\sigma)\right\|\right),
\end{gathered}
$$

where $\kappa_{i}$ is the eigenvalue condition number for NEPS,

$$
\kappa_{i}:=\frac{\left\|u_{i}\right\|\left\|v_{i}\right\|}{\left|u_{i}^{H} M^{\prime}\left(\lambda_{i}\right) v_{i}\right|},
$$

and $R_{\Gamma}(\sigma)$ is the remainder term in (4.5).

Proof The result follows the steps in (4.2) but instead using the form (4.5) and matrix norm inequalities.

\subsection{Characterization of Keldysh's remainder term $R_{\Gamma}(\sigma)$}

The analysis in the previous subsection indicates that a dependence on the eigenvalue clustering similar to the linear case can be expected under the condition that $R_{\Gamma}$ is small. Keldysh's theorem is, in a certain sense, a matrix version of the partial fraction expansion of an analytic function (also known as Mittag-Leffler's theorem) and has 
been characterized in [10]. See [45] for a partial fraction expansion characterization for delay eigenvalue problems. A precise characterization of $R_{\Gamma}$ and of its norm for the general case using [10] is somewhat involved. We take a less ambitious approach and point out certain situations where it is small or vanishes. Although $R_{\Gamma}$ does not always vanish in the limit where $\Gamma$ encloses $\mathbb{C}$, it does vanish under certain assumptions. We characterize several of such situations next and note that these results are general for nonlinear eigenvalue problems. Therefore, they may be of interest also outside the scope of quasi-Newton methods.

First, we need an explicit formulation of the remainder term $R_{\Gamma}$.

Lemma 4.2 Let $\Gamma \subset \Omega$ be a simple, closed, piecewise-smooth curve and denote the eigenvalues of (1.1) in the interior of $\Gamma$ by $\lambda_{1}, \cdots, \lambda_{k}$ and suppose they are simple. Then, the analytic function $R_{\Gamma}(z)$, i.e., the analytic part of $M(z)^{-1}$ in $\operatorname{int}(\Gamma) /\left\{\lambda_{1}, \ldots, \lambda_{k}\right\}$ given by the Keldysh theorem, has the integral representation

$$
R_{\Gamma}(z)=\frac{1}{2 \pi \mathrm{i}} \int_{\Gamma} \frac{M(\lambda)^{-1}}{\lambda-z} \mathrm{~d} \lambda .
$$

Proof We can apply the Cauchy integral formula, since $R_{\Gamma}$ is analytic, and use (4.5)

$$
\begin{aligned}
R_{\Gamma}(z) & =\frac{1}{2 \pi \mathrm{i}} \int_{\Gamma} \frac{R_{\Gamma}(z)}{\lambda-z} \mathrm{~d} \lambda \\
& =\frac{1}{2 \pi \mathrm{i}}\left(\int_{\Gamma} \frac{M(\lambda)^{-1}}{\lambda-z} \mathrm{~d} \lambda-\sum_{i=1}^{k} \frac{v_{i} u_{i}^{H}}{u_{i}^{H} M^{\prime}\left(\lambda_{i}\right) v_{i}} \int_{\Gamma} \frac{1}{\left(\lambda-\lambda_{i}\right)(\lambda-z)} \mathrm{d} \lambda\right) .
\end{aligned}
$$

The Cauchy residue theorem implies that $\int_{\Gamma} \frac{1}{\left(\lambda-\lambda_{i}\right)(\lambda-z)} \mathrm{d} \lambda=0$, which gives (4.6).

This leads directly to a sufficient condition for vanishing $R_{\Gamma}$, involving $M(\lambda)^{-1}$ in the limit $\lambda \rightarrow \infty$.

Lemma 4.3 Suppose $M(\lambda)$ is analytic in $\mathbb{C}$ and suppose all eigenvalues are simple. Moreover, suppose

$$
\left\|M(\lambda)^{-1}\right\| \rightarrow 0, \quad \text { as } \quad|\lambda| \rightarrow \infty
$$

Then, the set of eigenvalues is finite and

$$
M(\lambda)^{-1}=\sum_{i=1}^{k} \frac{1}{\lambda-\lambda_{i}} \frac{v_{i} u_{i}^{H}}{u_{i}^{H} M^{\prime}\left(\lambda_{i}\right) v_{i}}
$$

for all $\lambda \in \mathbb{C} \backslash\left\{\lambda_{1}, \lambda_{2}, \ldots, \lambda_{k}\right\}$, where $\lambda_{i}, 1 \leq i \leq k$, denote the eigenvalues of $M$.

Proof Since (4.7) implies that for every $\varepsilon>0$ there exists an $R$ such that $\sup _{|z|>R}\left\|M(z)^{-1}\right\|<\varepsilon$, we have in particular that there exists $r$ such that $M(z)^{-1}$ has no poles outside a disk of radius $r$. The eigenvalues of (1.1) are roots of the analytic function $\operatorname{det}(M(\lambda))$. An analytic function has only a finite number of roots in a compact subset of the complex plane, and we therefore only have a finite number of 
eigenvalues in the disk of radius $r$. Take $\Gamma$ to be a circle of radius $r$. Then, using the representation given by Lemma 4.2, we get the bound

$$
\left\|R_{\Gamma}(z)\right\| \leq \frac{1}{2 \pi} \int_{\Gamma} \frac{\| M(\lambda)^{-1}||}{|\lambda-z|} \mathrm{d} \lambda \leq \max _{z \in \Gamma}\left\|M(z)^{-1}\right\| .
$$

Letting $r \rightarrow \infty$, the claim follows.

Remark 4.2 (Generalizations to higher order multiplicities) Lemma 4.2 and Lemma 4.3 have the assumption that the eigenvalues are simple. A generalization to higher algebraic and/or geometric multiplicities seems feasible but more involved. Then, the expression (4.6) follows from [3, Corollary 2.8], and the fact that when the contour $\Gamma$ encircles $\lambda_{i}$ and $z$, and $\ell \geq 1$, it holds that

$$
\frac{1}{2 \pi i} \int_{\Gamma} \frac{1}{\left(\lambda-\lambda_{i}\right)^{\ell}(\lambda-z)} \mathrm{d} \lambda=0
$$

by the residue theorem. Then, the condition ||$M(\lambda)^{-1}|| \rightarrow 0$ as $|\lambda| \rightarrow \infty$ and the inequality (4.9) imply that $R_{\Gamma}(z)=0$.

From the above lemmas, we conclude the following result which states that the $R_{\Gamma}$ vanishes if the NEP is the sum of a polynomial with a non-singular leading coefficient matrix and a term which decays sufficiently fast.

Theorem 4.4 Suppose $M$ is analytic in $\mathbb{C}$ and suppose all eigenvalues are simple. Moreover, suppose $M(\lambda)$ is of the form

$$
M(\lambda)=P(\lambda)+F(\lambda),
$$

where

1. $P(\lambda)=\sum_{i=0}^{N} A_{i} \lambda^{i}$ for some matrices $A_{0}, \ldots, A_{N} \in \mathbb{C}^{n \times n}$ such that $A_{N}$ is non-singular, and

2. $\lambda^{-N} F(\lambda) \rightarrow 0$ as $|\lambda| \rightarrow \infty$.

Then, $R_{\Gamma}(\lambda)=0$ and the representation (4.8) hold for $M(\lambda)^{-1}$.

Proof We see that

$$
M(\lambda)^{-1}=\left(\sum_{\ell=0}^{N} \lambda^{\ell} A_{\ell}+F(\lambda)\right)^{-1}=\frac{A_{N}^{-1}}{\lambda^{N}}(I+B(\lambda))^{-1},
$$

where $B(\lambda)=\left(\sum_{\ell=0}^{N-1} \lambda^{\ell-N} A_{\ell}+\lambda^{-N} F(\lambda)\right) A_{N}^{-1}$. By assumption 2 above, it holds that

$$
\begin{aligned}
\|B(\lambda)\| & =\left\|\left(\sum_{\ell=0}^{N-1} \lambda^{\ell-N} A_{\ell}+\lambda^{-N} F(\lambda)\right) A_{N}^{-1}\right\| \\
& \leq\left\|A_{N}^{-1}\right\|\left(\sum_{\ell=0}^{N-1}|\lambda|^{\ell-N}\left\|A_{\ell}\right\|+\left\|\lambda^{-N} F(\lambda)\right\|\right) \rightarrow 0, \quad \text { as } \quad|\lambda| \rightarrow \infty .
\end{aligned}
$$


Using the bound $\left\|(I-A)^{-1}\right\| \leq(1-\|A\|)^{-1}$ for $\|A\|<1$ (see [13, pp. 351]), and choosing $\lambda$ such that $\|B(\lambda)\|<1$, we see from (4.10) that

$$
\left\|M(\lambda)^{-1}\right\| \leq \frac{\left\|A_{N}^{-1}\right\|}{|\lambda|^{N}} \frac{1}{1-\|B(\lambda)\|}
$$

and therefore $\left\|M(\lambda)^{-1}\right\| \rightarrow 0, \quad$ as $\quad|\lambda| \rightarrow \infty$.

In addition to the polynomial eigenvalue problem with an invertible leading coefficient matrix, the conditions of Lemma 4.3 (and the representation (4.8), subsequently) hold for several problems in the literature. For instance, the following class of rational eigenvalue problems are often encountered in practice [2]. Let $A, B$, and $C_{i}, 1 \leq i \leq k$, be square matrices such that $B$ is invertible, and let

$$
M(\lambda)=A+\lambda B+\sum_{i=1}^{k} \frac{\lambda}{\sigma_{i}-\lambda} C_{i}
$$

where $\sigma_{i}$ are given poles.

These results hold also for certain modifications of the symmetric eigenvalue problem; see, e.g., [14] and [33]. Let $A, B \in \mathbb{C}^{n \times n}$ be such that $B$ is invertible, and let $s: \mathbb{C} \rightarrow \mathbb{C}$ be a function such that $s(\lambda) \rightarrow C, C$ constant, as $|\lambda| \rightarrow \infty$. Let

$$
M(\lambda)=A-\lambda B+s(\lambda) u u^{H}
$$

The condition on the limit behavior of $s(\lambda)$ holds in particular for [14, Example 2].

Remark 4.3 (A counterexample) Although the above theory shows that $R_{\Gamma}$ does vanish in many situations similar to the linear case, it is not always zero, as can be seen from the example

$$
M(\lambda)=\left[\begin{array}{cc}
\lambda-1 & (\lambda-1)(\lambda-2) f(\lambda) \\
0 & \lambda-2
\end{array}\right]
$$

where $f$ is a scalar analytic function. Then,

$$
M(\lambda)^{-1}=\left[\begin{array}{cc}
(\lambda-1)^{-1} & f(\lambda) \\
0 & (\lambda-2)^{-1}
\end{array}\right]
$$

and when we select $\Gamma$ such that encloses the two eigenvalues $\lambda_{1}=1$ and $\lambda_{2}=2$, we have

$$
R_{\Gamma}(z)=\frac{1}{2 \pi \mathrm{i}} \int_{\Gamma} \frac{M(\lambda)^{-1}}{\lambda-z} \mathrm{~d} \lambda=\left[\begin{array}{cc}
0 & f(z) \\
0 & 0
\end{array}\right] \quad \text { for all } z \in \mathbb{C} \backslash\{1,2\}
$$




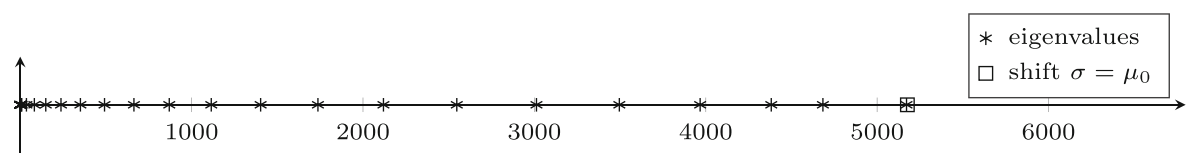

Fig. 1 Spectrum of the rational eigenvalue problem (5.1)

\section{Numerical simulations}

\subsection{Rational eigenvalue problem}

We illustrate several theoretical properties of the methods with numerical simulations. ${ }^{1}$ We first consider the problem "loaded string" from the NLEVP collection [2]. The problem is of the form

$$
M(\lambda)=A-\lambda B+\frac{\lambda}{\lambda-\sigma} C,
$$

where $A, B, C \in \mathbb{R}^{n \times n}, B$ invertible. We set $n=20$. All the eigenvalues are real positive, and to make the spectrum more clustered in the left end, we multiply the original coefficient matrix $C$ by $n$. The spectrum of the problem is shown in Fig. 1 .

We first place the initial value $\left(\mu_{0}, x_{0}\right)$ close to the right-most eigenvalue $\lambda \approx$ 5170 , such that $\mu_{0}=\lambda+5.0$ and $x_{0}=v+a \cdot[1 \ldots 1]^{T}$, where $v$ is the exact eigenvector corresponding to $\lambda$ and $a$ is a scalar. We set $c=x_{0}$ and $\sigma=\mu_{0}$. Since we are here mainly concerned with convergence properties, we use computation with high precision arithmetic with a sufficiently high precision, such that round-off errors are not influencing the figures. As shown in Fig. 2, the performance of QN1 is found to be sensitive to the distance of $x_{0}$ from $v$. As expected from Corollary 3.3, the convergence curves of the quasi-Newton 2 and residual inverse iteration are very close to each other. Figure 3 shows the numerically estimated convergence factors and also the a priori convergence factor estimates. For QN1, the a priori converge factor equals $\rho\left(A_{1}\right)$, i.e., the spectral radius of the matrix $A_{1}$ given in Theorem 3.1. For QN2 and QN3, i.e., residual inverse iteration, it equals $\rho(B)$, where $B$ is given in Corollary 3.3. The estimated convergence factor $\rho_{k}$ at iteration $k$ is computed by

$$
\rho_{k}=\frac{\left\|w_{k}-w_{*}\right\|}{\left\|w_{k-1}-w_{*}\right\|},
$$

where $w_{k}=\left[\begin{array}{c}v_{k} \\ \mu_{k}\end{array}\right]$ and $w_{*}$ denote the exact solution $w_{*}=\left[\begin{array}{l}v \\ \lambda\end{array}\right]$.

Then, we consider the initial value $\left(\mu_{0}, x_{0}\right)$ close to a left-end eigenvalue $\lambda \approx$ 9.07, such that again $\mu_{0}=\lambda+5.0, x_{0}=v+a \cdot\left[\begin{array}{lll}1 & \ldots & 1\end{array}\right]^{T}(a>0)$, and $c=x_{0}$ and $\sigma=\mu_{0}$. As can be expected from the bound given in Corollary 4.1, the convergence is now slower since $\lambda$ is in a cluster of eigenvalues. This is depicted by Fig. 4. Again, the performance of QN1 is found to be sensitive to the distance of $x_{0}$ from $v$.

As shown in Fig. 4, the convergence is now slower than for the right-most eigenvalue, as is expected from the clustering of the spectrum depicted in Fig. 1,

\footnotetext{
${ }^{1}$ The simulations are publicly available online: http://www.math.kth.se/ eliasj/src/qnewton/.
} 


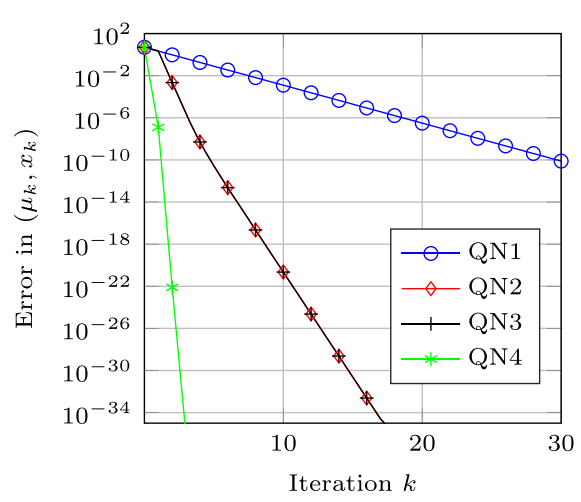

(a) $a=0.15$

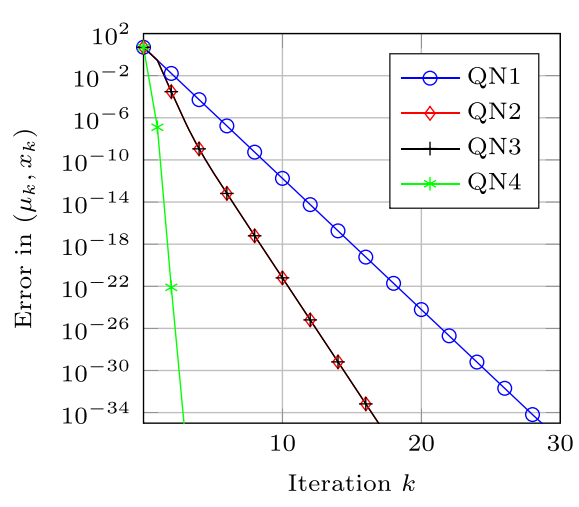

(b) $a=0.05$

Fig. 2 The convergence of the three different methods when (a) $c=x_{0}$ is further from $(a=0.15)$ and (b) closer to $(a=0.05)$ the exact eigenvector $v$

\subsection{Quadratic eigenvalue problem}

In this section, we illustrate the influence of the eigenvalue clustering on the convergence factor. The bounds for the convergence factor illustrating this effect were derived in Section 3 and can be clearly identified from the following example. Consider the quadratic eigenvalue problem

$$
M(\lambda)=\lambda^{2} I-\lambda\left(A_{1}+A_{2}\right)+A_{1} A_{2},
$$

where $A_{1}, A_{2} \in \mathbb{C}^{10 \times 10}$ are diagonal matrices. The set eigenvalues of this problem is the union of eigenvalues $A_{1}$ and $A_{2}$. We choose the diagonal elements of $A_{1}$ and $A_{2}$ such that eigenvalues of $M(\lambda)$ are 0.1 and 19 equally distributed points on the circle of radius $r$, as illustrated in Fig. 5 for $r=0.5$.
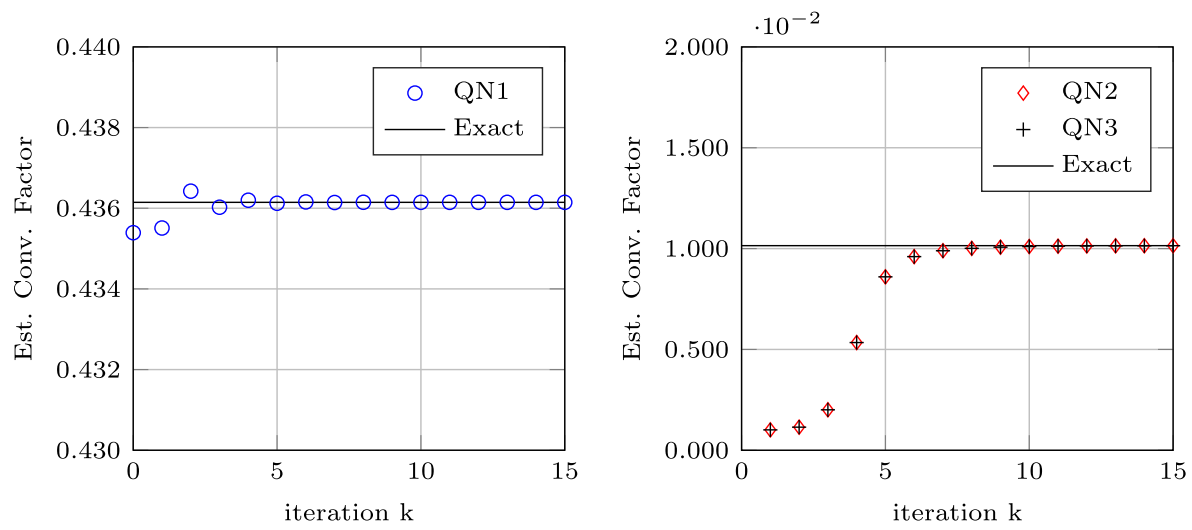

Fig. 3 Estimated convergence factors of the three different methods. The solid line is the a priori computed spectral radius of $A$ in Corollary 3.3 and the dashed line that of Theorem 3.1 


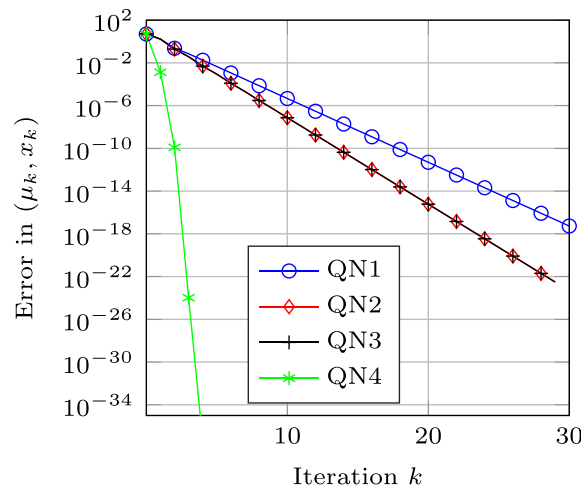

(a) $a=0.2$

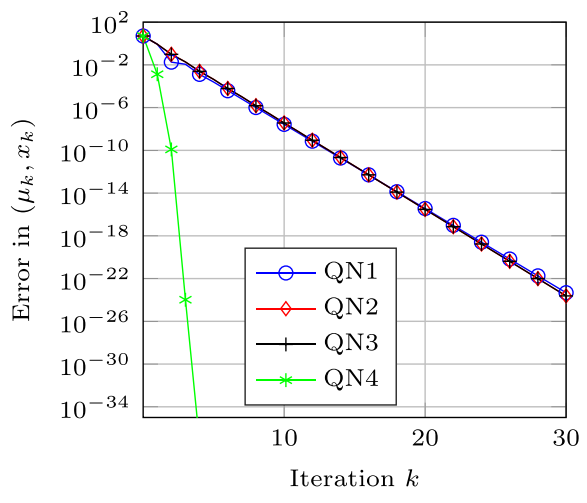

(b) $a=0.1$

Fig. 4 The convergence of the three different methods when (a) $c=x_{0}$ is further from $(a=0.2)$ and (b) closer to $(a=0.1)$ the exact eigenvector $v$

We first place the initial eigenpair approximation $\left(\mu_{0}, x_{0}\right)$ to the origin, such that $\mu_{0}=0$ and $x_{0}=v+0.1 \cdot[1 \ldots 1]^{T}$, where $v$ is the exact eigenvector corresponding to the eigenvalue 0.1 . We set $c=x_{0}$ and $\sigma=\mu_{0}$. Figure 6 shows the convergence of the methods and also the numerically estimated convergence factors for the three first quasi-Newton methods. QN1 is found again to have the slowest convergence, and the a priori convergence factor estimates computed using Theorem 3.1 and Corollary 3.3 are again found to be sharp.

Let $\rho_{r}$ be the spectral radius of the iteration matrix $B$ given in Corollary 3.3, i.e., the convergence factor for QN2 and QN3. In Fig. 7, we illustrate how $\rho_{r}$ behaves as a function of $r$, the radius of the circle. The value of $\rho_{r}$ is computed for 10 different values of $r$ varying from $10^{-1 / 2}$ to $10^{5}$. As expected from Corollary 3.3, we observe that $\rho_{r} \sim 1 / r$.

\subsection{A large-scale problem}

We now consider a large-scale NEP which arises in the study of waves traveling in a periodic medium $[16,36]$. More precisely, the waveguide eigenvalue problem,

Fig. 5 Spectrum of the quadratic problem (5.2) when $r=0.5$

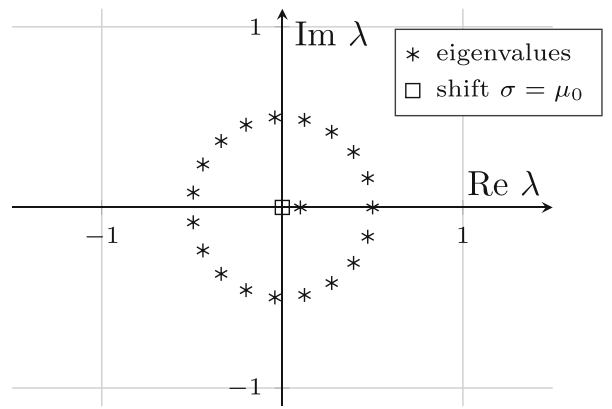




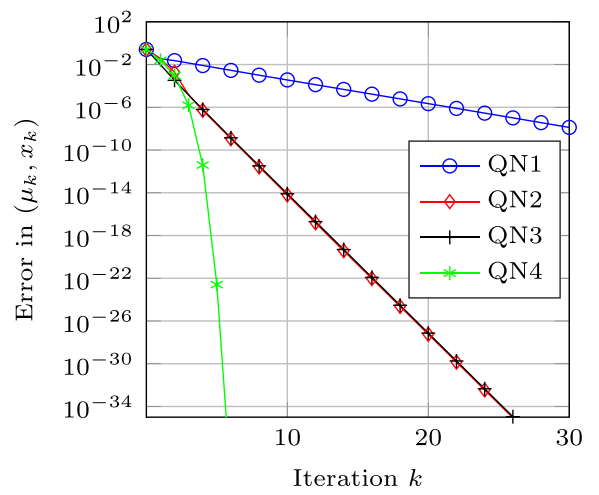

(a) $r=0.5$

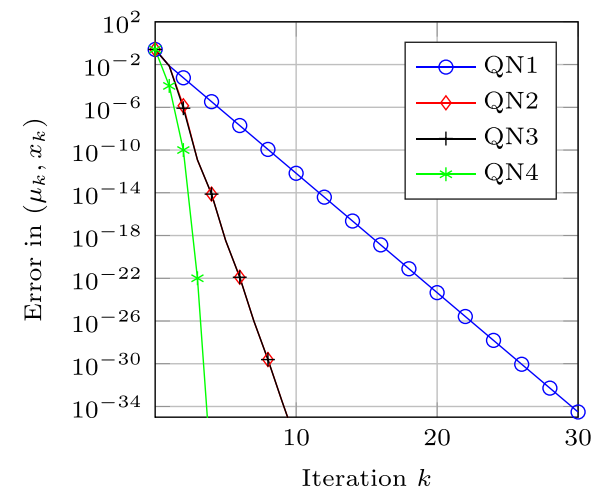

(b) $r=100$

Fig. 6 Convergence of the four different methods for the quadratic problem (5.2) when (a) $r=0.5$ and (b) $r=100$

without the Cayley transformation, associated to the waveguide described in [16, Section 5.2] is considered. The problem is formulated as

$$
M(\lambda)=\left(\begin{array}{cc}
Q(\lambda) & C_{1}(\lambda) \\
C_{2}^{T} & P(\lambda)
\end{array}\right) .
$$

We choose the discretization parameters $n_{x}=200$ and $n_{z}=201$, which means that the size of the NEP is $n=n_{x} n_{z}+2 n_{z}=40602$. The matrix $C_{2}^{T}$ and the second degree polynomials $Q(\lambda)$ and $C_{1}(\lambda)$ are sparse, and the matrix $P(\lambda)$ is dense and it is defined by nonlinear functions of $\lambda$ involving square roots of polynomials. The matrix-vector product $P(\lambda) w$ is efficiently computed using two Fast Fourier Transforms (FFTs) and a multiplication with a diagonal matrix. The linear systems

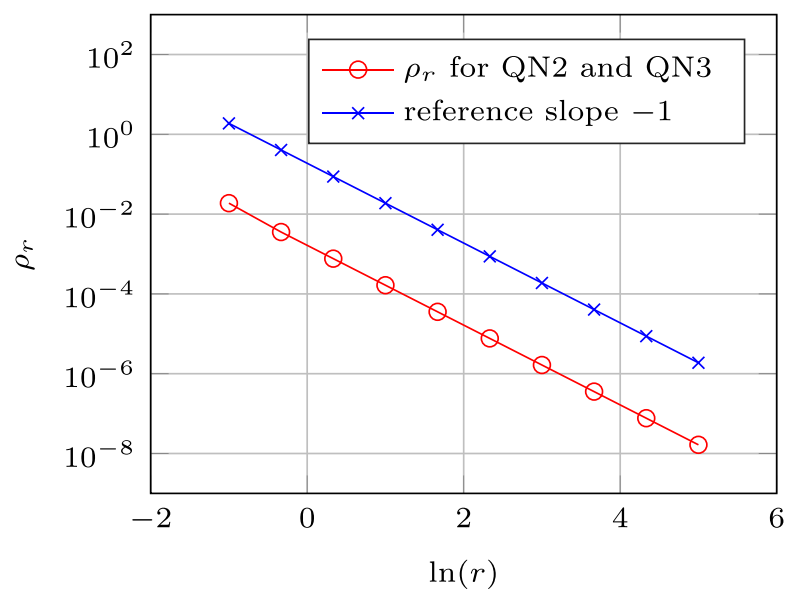

Fig. 7 Plot of the convergence factor $\rho_{r}$ versus $r$ for QN2 and the quadratic problem (5.2) 


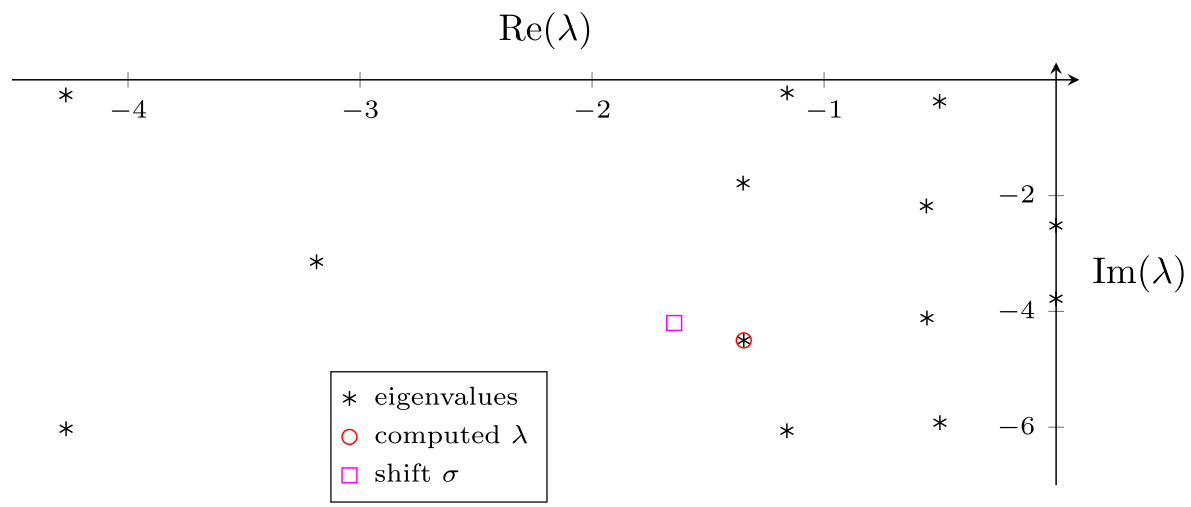

Fig. 8 Spectrum of the waveguide eigenvalue problem described in [16, Section 5.2]

involving the matrix $M(\sigma)$ can be solved by precomputing a Schur complement. See [16] for a full description of the problem.

We compare QN1, QN2, and QN3 for approximating a specific eigenpair. The pair $(\lambda, v)$ denotes the accurate approximation of the wanted eigenpair. The shift $\sigma$ is selected close to the wanted eigenvalue $\lambda$, more precisely, $|\sigma-\lambda| \approx 0.42$ (see Fig. 8), and the initial guess $x_{0}$ is selected such that $\left\|x_{0}-v\right\| \approx 10^{-5}$. The error is computed as the absolute value of the distance between the wanted eigenvalue and the current eigenvalue approximation, namely $\left|\mu_{k}-\lambda\right|$. If an initial guess of the eigenvector is provided, all the methods present similar convergence rate and QN2 and QN3 are slightly faster then QN1 (see Fig. 9). The block $(1,2)$ of the matrix $A_{1}$ defined in (3.2) has norm approximatively $10^{-3}$. This may suggest that the spectral radius of this matrix is close to the spectral radius of $B$ given in (3.6). In particular, the convergence rate of QN1 is expected to be close to the convergence rate of QN2 and QN3. This is consistent with the numerical simulation. With a random initial guess $x_{0}$ of the eigenvector, QN1 does not converge whereas QN2 and QN3 still converge with the same convergence rate but with they require more iterations since the initial

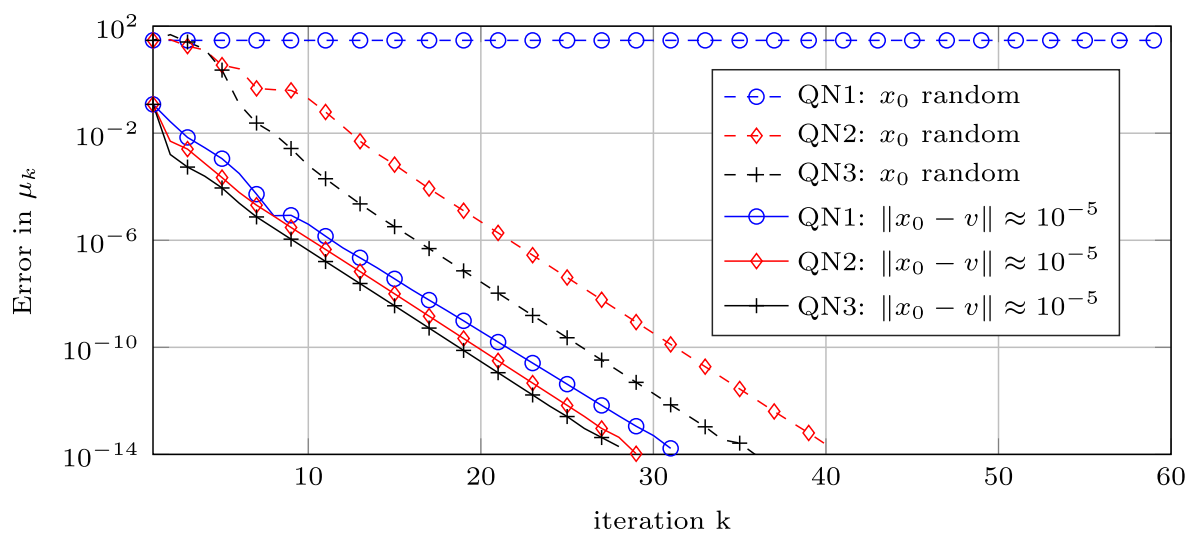

Fig. 9 Convergence of QN1, QN2, and QN3 for computing the eigenvalue $\lambda$ 
error is larger. See Fig. 9. This can be justified observing that, in this case, the block $(1,2)$ of the matrix $A_{1}$ defined in (3.2) has norm approximatively $10^{-1}$. Therefore, the convergence factor of QN1 is expected to be significantly different from QN2 and QN3 by using the previous reasoning.

\section{Conclusions}

We have here presented four iterative methods and showed how they can be analyzed with techniques of quasi-Newton methods and Keldysh's theorem. We have also illustrated how two well-established methods can be interpreted as quasi-Newton methods. A secondary conclusion that we wish to stress here is that, in general, methods of this type can in an insightful way be analyzed in the framework of quasiNewton methods. We have illustrated how linear as well as higher order convergence can be shown in this framework. There are many results for quasi-Newton methods which can potentially be applied to these methods, e.g., techniques which improve the convergence basin [9, 11], scaling techniques [6], adaption for non-smooth (or almost non-smooth) problems [24], and general convergence results [44].

The method corresponding to keeping only the $(1,1)$-block constant, i.e., (1.7), is to our knowledge a new method in the context of NEPs. Moreover, although it has several similarities with residual inverse iteration in terms of the convergence and in terms of the behavior for linear problems, it can be in several situations more attractive than residual inverse iteration. Note that unlike QN2, residual inverse iteration requires solving a nonlinear scalar problem. For some NEPs, the action of $M(\lambda)$ is only implicitly available, e.g., in the form of a differential equation as in [28]. Therefore, the computation of a solution to the scalar nonlinear (2.9b) is computationally more demanding than computing the product $M^{\prime}(\mu) r$ which is required in $(2.8 \mathrm{~b})$.

We have in this paper considered specific variants of quasi-Newton methods by keeping blocks of the Jacobian matrix constant or making them implicit. The presented methods do not correspond to all variants of constant and implicit constructions. We have considered many other variants of quasi-Newton methods, most of which are neither of theoretical or practical value and have therefore been omitted.

We do have a negative conclusion in this paper. We have concluded that QN1 does not appear very competitive in practice since better convergence is usually achieved from QN2, although QN1 is based on the most common quasi-Newton approach, i.e., keeping the Jacobian matrix constant. There is another important Newton-based algorithm which we have not considered in this manuscript, the Jacobi-Davidson algorithm ([30, 32]). Although the Jacobi-Davidson algorithm does have interpretations in terms of Newton's method as pointed out, e.g., in [31, Section 6], our results here are not directly applicable. In [31, Section 6], the authors point out that the correction equation of the Jacobi-Davidson algorithm can be derived from Newton's method on the nonlinear equation $G(x)=M(p(x)) x$ where $p(x)$ is the Rayleigh functional. Note that this nonlinear equation is different from the equation underlying our augmented system (1.3). Moreover, the fact that the solution of this nonlinear equation is a manifold and the solution (eigenvector) is not isolated in the standard sense prevents us from directly applying results for quasi-Newton methods. 
Finally, we wish to specifically stress that the presented results may be of considerable value beyond the scope of this article. Several of the methods presented here form the basis of other state-of-the-art methods such as the subspace accelerated extensions of residual inverse iteration (the nonlinear Arnoldi method [39]), preconditioned versions [8] and inner-outer-iteration constructions as in [43]. Those extensions may possibly also be interpreted in a quasi-Newton setting, but this extension would require attention beyond this manuscript.

Acknowledgements We thank Wim Michiels (KU Leuven) for valuable discussions regarding partial fraction expansions for time-delay systems.

Open Access This article is distributed under the terms of the Creative Commons Attribution 4.0 International License (http://creativecommons.org/licenses/by/4.0/), which permits unrestricted use, distribution, and reproduction in any medium, provided you give appropriate credit to the original author(s) and the source, provide a link to the Creative Commons license, and indicate if changes were made.

\section{References}

1. Van Beeumen, R., Meerbergen, K., Michiels, W.: A rational Krylov method based on Hermite interpolation for nonlinear eigenvalue problems. SIAM J. Sci. Comput. 35(1), A327-A350 (2013)

2. Betcke, T., Higham, N.J., Mehrmann, V., Schröder, C., Tisseur, F.: NLEVP A collection of nonlinear eigenvalue problems. ACM Trans. Math. Softw. 39(2), 1-28 (2013)

3. Beyn, W.-J.: An integral method for solving nonlinear eigenvalue problems. Linear Algebra Appl. 436(10), 3839-3863 (2012)

4. Beyn, W.-J., Effenberger, C., Kressner, D.: Continuation of eigenvalues and invariant pairs for parameterized nonlinear eigenvalue problems. Numer. Math. 119(3), 489-516 (2011)

5. Dembo, R.S., Eisenstat, S., Steihaug, T.: Newton inexact methods. SIAM J. Numer. Anal. 19, 400-408 (1982)

6. Deuflhard, P.: Newton Methods for Nonlinear Problems: Affine Invariance and Adaptive Algorithms, vol. 35. Springer (2011)

7. Effenberger, C.: Robust Solution Methods for Nonlinear Eigenvalue Problems. PhD thesis, EPF Lausanne (2013)

8. Effenberger, C., Kressner, D.: On the residual inverse iteration for nonlinear eigenvalue problems admitting a Rayleigh functional. Technical report, EPF Lausanne (2014)

9. Eisenstat, S.C., Walker, H.F.: Globally convergent inexact Newton methods. SIAM J. Optim. 4(2), 393-422 (1994)

10. Gohberg, I., Rodman, L.: Interpolation and local data for meromorphic matrix and operator functions. Integr. Equ. Oper. Theory 9(1), 60-94 (1986)

11. González-Lima, M., de Oca, F.N.: A Newton-like method for nonlinear system of equations. Numer. Algor. 52(3), 479 (2009)

12. Homeier, H.H.: A modified Newton method for rootfinding with cubic convergence. J. Comput. Appl. Math. 157(1), 227-230 (2003)

13. Horn, R., Johnson, C.: Matrix Analysis. Cambridge University Press, Cambridge (2012)

14. Huang, X., Bai, Z., Su, Y.: Nonlinear rank-one modification of the symmetric eigenvalue problem. J. Comput. Appl Math. 28(2), 218-234 (2010)

15. Jarlebring, E.: Convergence factors of Newton methods for nonlinear eigenvalue problems. Linear Algebra Appl. 436(10), 3943-3953 (2012)

16. Jarlebring, E., Mele, G., Runborg, O.: The waveguide eigenvalue problem and the tensor infinite Arnoldi method. SIAM J. Sci. Comput. 39, A1062-A1088 (2017)

17. Jarlebring, E., Michiels, W.: Analyzing the convergence factor of residual inverse iteration. BIT 51(4), 937-957 (2011)

18. Kou, J.: The improvements of modified Newton's method. Appl. Math. Comput. 189(1), 602-609 (2007) 
19. Kressner, D.: A block Newton method for nonlinear eigenvalue problems. Numer. Math. 114(2), 355-372 (2009)

20. Lancaster, P.: Lambda-Matrices and Vibrating Systems. In: International Series of Monographs in Pure and Applied Mathematics, vol. 94 (1966)

21. Van Loan, C.h.: On estimating the condition of eigenvalues and eigenvectors. Linear algebra Appl. 88, 715-732 (1987)

22. Mehrmann, V., Voss, H.: Nonlinear eigenvalue problems: a challenge for modern eigenvalue methods. GAMM-Mitt. 27, 121-152 (2004)

23. Mennicken, R., Möller, M.: Non-self-adjoint Boundary Eigenvalue Problems, vol. 192. Gulf Professional Publishing (2003)

24. Mietański, M.J.: Convergence of an inexact generalized Newton method with a scaled residual control. Comput. Math. Appl. 61(6), 1624-1632 (2011)

25. Neumaier, A.: Residual inverse iteration for the nonlinear eigenvalue problem. SIAM J. Numer. Anal. 22, 914-923 (1985)

26. Osborne, M., Michaelson, S.: The numerical solution of eigenvalue problems in which the eigenvalue parameter appears nonlinearly, with an application to differential equation. Comput. J. 7, 66-71 (1964)

27. Peters, G., Wilkinson, J.: Inverse iterations, ill-conditioned equations and Newton's method. SIAM Rev. 21, 339-360 (1979)

28. Rott, O., Jarlebring, E.: An iterative method for the multipliers of periodic delay-differential equations and the analysis of a PDE milling model. In Proceedings of the 9th IFAC Workshop on Time-delay Systems. Prague, pp 1-6 (2010)

29. Ruhe, A.: Algorithms for the nonlinear eigenvalue problem. SIAM J. Numer. Anal. 10, 674-689 (1973)

30. Schreiber, K.: Nonlinear Eigenvalue Problems: Newton-Type Methods and Nonlinear Rayleigh Functionals. PhD thesis TU, Berlin (2008)

31. Sleijpen, G., Van der Vorst, H.A.: The Jacobi-Davidson method for eigenvalue problems and its relation with accelerated inexact Newton scheme. In: Proceedings of the Second IMACS International Symposium on Iterative Methods in Linear Algebra IMACS (2006)

32. Sleijpen, G.L., Booten, A.G., Fokkema, D.R., van der Vorst, H.A.: Jacobi-Davidson type methods for generalized eigenproblems and polynomial eigenproblems. BIT 36(3), 595-633 (1996)

33. Solovëv, S.I.: Preconditioned iterative methods for a class of nonlinear eigenvalue problems. Linear algebra Appl. 415(1), 210-229 (2006)

34. Szyld, D.B., Xue, F.: Local convergence analysis of several inexact newton-type algorithms for general nonlinear eigenvalue problems. Numer. Math. (2012)

35. Szyld, D.B., Xue, F.: Several properties of invariant pairs of nonlinear algebraic eigenvalue problems. IMA J. Numer. Anal. 34(3), 921-954 (2014)

36. Tausch, J., Butler, J.: Floquet multipliers of periodic waveguides via Dirichlet-to-Neumann maps. J. Comput. Phys. 159(1), 90-102 (2000)

37. Unger, G.: Convergence orders of iterative methods for nonlinear eigenvalue problems. In: Advanced Finite Element Methods and Applications. Springer, pp. 217-237 (2013)

38. Unger, H.: Nichtlineare Behandlung von Eigenwertaufgaben. Z. Angew. Math. Mech. 30, 281-282 (1950). English translation: http://www.math.tu-dresden.de/ $\sim$ schwetli/Unger.html

39. Voss, H.: An Arnoldi method for nonlinear eigenvalue problems. BIT 44, 387-401 (2004)

40. Voss, H.: Nonlinear eigenvalue problems. In: Hogben, L. (ed.) Handbook of Linear Algebra, Second Edition, number 164 in Discrete Mathematics and its Applications. Chapman and Hall/CRC (2013)

41. Voss, H., Werner, B.: A minimax principle for nonlinear eigenvalue problems with applications to nonoverdamped systems. Math. Methods Appl. Sci. 4, 415-424 (1982)

42. Werner, B.: Das Spektrum von Operatorenscharen mit verallgemeinerten Rayleighquotienten. $\mathrm{PhD}$ thesis, Fachbereich Mathematik Universitt Hamburg (1970)

43. Xue, F., Szyld, D.B.: Efficient preconditioned inner solves for inexact Rayleigh quotient iteration and their connections to the single-vector jacobi-davidson method. Technical report (2011)

44. Ypma, T.J.: Local convergence of inexact Newton methods. SIAM J. Numer. Anal. 21(3), 583-590 (1984)

45. Zwart, H.J., Curtain, R.F., Partington, J.R., Glover, K.: Partial fraction expansions for delay systems. Syst. Control Lett. 10(4), 235-243 (1988) 